EPJ Web of Conferences 59, 02003 (2013)

DOI: $10.1051 /$ epjconf/20135902003

(C) Owned by the authors, published by EDP Sciences, 2013

\title{
Some recent studies on hohlraum physics
}

\author{
Ke Lana , Wen Yi Huo, Xin Li, Guoli Ren, Yongsheng Li, Xujun Meng, \\ Changshu Wu, Shiyang Zou, Xiumei Qiao, Peijun Gu, Wudi Zheng, \\ Dongxian Lai and Tinggui Feng
}

Institute of Applied Physics and Computational Mathematics, PO Box 8009-14 Beijing, 100088, PR China

\begin{abstract}
Some of our recent studies on hohlraum physics are presented, mainly including simulation study on hohlraum physics experiments on SGIII prototype, the design of $\mathrm{Au}+\mathrm{U}+\mathrm{Au}$ sandwich hohlraum for ignition target, and an initial design of elliptical hohlraum and pertinent drive laser power in order to generate an ignition radiation profile.
\end{abstract}

\section{INTRODUCTION}

Hohlraum plays a key role in the study of indirect drive inertial fusion [1,2], and worldwide IFE (Inertial Fusion Energy) scientists have been paying many efforts in the study of hohlraum physics [3, 4]. Here, we will present some of our recent studies on hohlraum energetic and hohlraum design, including studies on recent hohlraum physics experiments on SGIII prototype [5], $\mathrm{Au}+\mathrm{U}+\mathrm{Au}$ sandwich hohlraum for ignition target [6], and method of giving an initial design of hohlraum and driving laser to generate a required radiation for 2-D optimizations [7]. In addition, we have also obtained some interesting results on x-ray ablative shock waves [8] and proposed a novel method for simultaneously determining the maximum radiation temperature and M-band fraction inside a hohlraum by using the shock wave technology [9], which was presented by Yongsheng Li, et al., in this conference.

\section{SIMULATION STUDY ON RECENT HOHLRAUM EXPERIMENTS ON SGIII PROTOTYPE}

We use our 2-D code LARED-H to study recent hohlraum experiments performed on SGIII-prototype laser facility. In the experiment, the laser pulse has a $1 \mathrm{~ns}$ flat top with $150 \mathrm{ps}$ rising and falling edges, and eight laser beams simultaneously irradiate hohlraum from two ends at an incidence cone of $45^{\circ}$ angle. The vacuum Au hohlraum used in experiments is $1000 \mu \mathrm{m}$ in diameter and $2100 \mu \mathrm{m}$ in length, with a $650 \mu \mathrm{m}$ diameter laser entrance hole (LEH) at each end. There are two broad-band soft x-ray spectrometers (SXSs) used for measuring the X-ray flux and spectrum emitted from the hohlraum, one measures from the LEH at an angle of $30^{\circ}$ to the hohlraum axis, and the other one measures along the line about $19^{\circ}$ from the normal direction of the diagnostic hole. We simulate and study the hohlraum energetics of these experiments and obtain the following results:

\footnotetext{
ae-mail: ke_lan68@yahoo.com
}

This is an Open Access article distributed under the terms of the Creative Commons Attribution License 2.0, which permits unrestricted use, distribution, and reproduction in any medium, provided the original work is properly cited. 


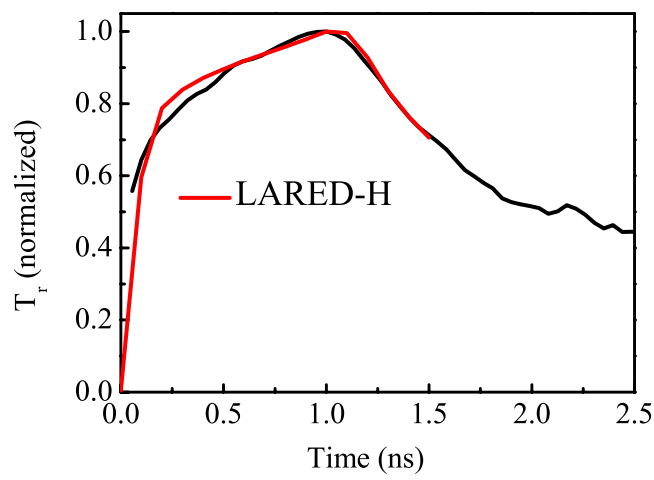

Figure 1. Comparison of the normalized temporal profiles of $\mathrm{Tr}$ from the simulation (red line) with that from the observation of SXS1 (black line).

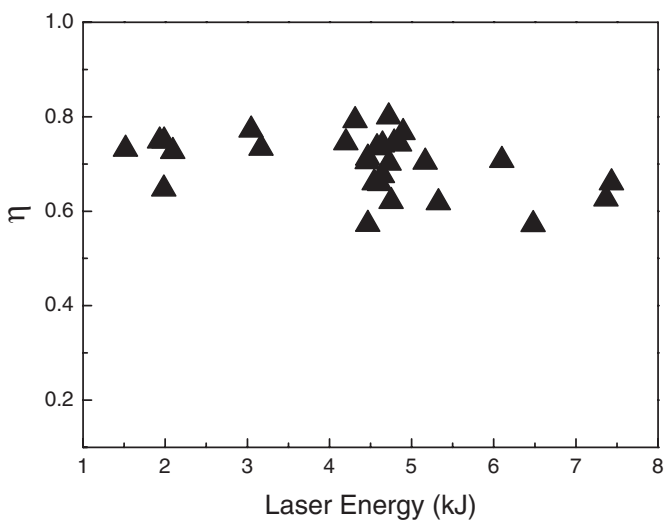

Figure 2. The coupling efficiencies of hohlraum under different laser energies evaluated from LARED-H simulations.

1. The temporal profile of $T_{r}$ from simulation agrees well with that from observations, except some discrepancies existing on the rising edge, as shown in Fig. 1. The laser energies of these shots used in comparison are around $5 \mathrm{~kJ}$, and the laser energy for simulation is taken as $5 \mathrm{~kJ}$.

2. From both simulation and observation, the emissions of $N$-band and $O$-band dominate the Hohlraum radiation. Moreover, the spectrum depends strongly on the observing direction.

3. The technologies of back-scattered light and hard $\mathrm{x}$-ray/hot electron measurements are under developing on SGIII prototype, we therefore calculate the coupling efficiency [10] by adjusting the laser energy so that the calculated peak radiation temperature is equal to the measured peak. As shown in Fig. 2, the coupling efficiency is around 70\%. Notice that the discrepancy of data shown in figure is somewhat large, which may be due to the fact that we do not use beam smoothing technology on SGIII-prototype, and thus it leads to an unstable backscatter refraction produced by the laser plasma interactions.

\section{STUDY ON AU + U + AU SANDWICH HOHLRAUM FOR 300 EV IGNITION TARGET}

In ignition targets designs, $U$ or $U$ based cocktail hohlraums [11] are usually used because the Rosseland mean opacity of $\mathrm{U}$ is higher than that of $\mathrm{Au}$ at the radiation temperature for ignition. However, it should be noticed that there is a longer than $10 \mathrm{~ns}$ prepulse at temperatures lower than 


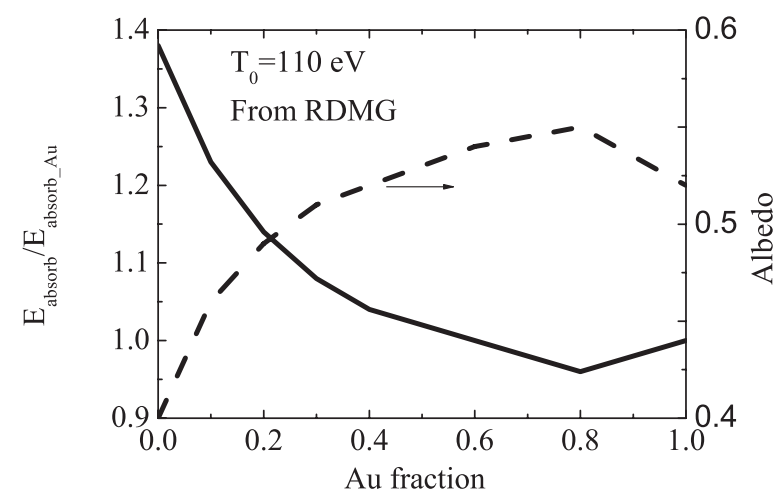

Figure 3. Ratios of albedo (dashed line) and energy absorption (solid line) in U-Au mixture to those in pure Au at $T_{0}=110 \mathrm{eV}$.

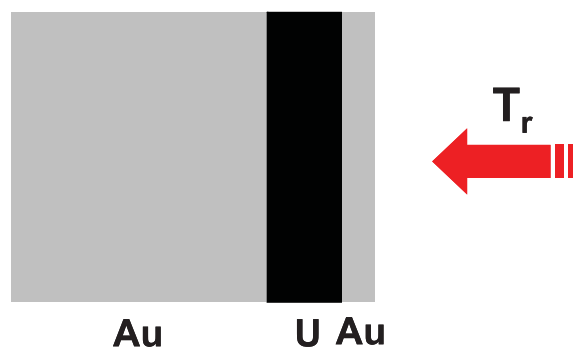

Figure 4. Schematic of the $(0.1 \sim 0.6 \mu \mathrm{m}) \mathrm{Au}+19.9 \mu \mathrm{m} \mathrm{U}+(\sim 5 \mu \mathrm{m})$ Au sandwich target in ignition target design.

$170 \mathrm{eV}$ in the radiation drive for ignition, while the opacity of $\mathrm{U}$ is actually lower than that of $\mathrm{Au}$ within this low temperature region. Shown in Fig. 3 is the ratio of energy absorptions (U-Au mixture over pure $\mathrm{Au}$ ) under radiation drive of $T_{0}=110 \mathrm{eV}$. As shown, the energy absorption of pure $\mathrm{U}$ relative to that of pure $\mathrm{Au}$ is near $140 \%$. The albedo of $\mathrm{U}-\mathrm{Au}$ mixture is also presented in the figure for comparison. In addition, the depth penetrated by radiation is only several micrometers under a $300 \mathrm{eV}$ radiation. We therefore proposed an $\mathrm{Au}+\mathrm{U}+\mathrm{Au}$ sandwich hohlraum for ignition targets in this work, shown in Fig. 4. The code we used for this study is RDMG, and the absorption cross section is calculated with our relativistic Hartree-Fock-Slater (HFS) self-consistent average atom model OPINCH.

We use RDMG simulation results to give the sandwich design for a typical National Ignition Facility (NIF) radiation drive of $300 \mathrm{eV}$ [2]. From our simulations, the energy absorption of an $\mathrm{Au}+\mathrm{U}$ target reaches its minimum when the inner Au layer is around $0.1 \mu \mathrm{m}$, but nevertheless its variation is small when the inner Au layer is within $0.6 \mu \mathrm{m}$. When the inner Au layer is thicker than $0.6 \mu \mathrm{m}$, the energy absorption increases seriously. Taking the inner Au layer as $0.1 \mu \mathrm{m}$, we further choose the optimum thickness of the medium $U$ layer by simulation. As a result, the optimum thickness of $\mathrm{U}$ layer is around $5 \mu \mathrm{m}$. The advantage of a sandwich target is almost the same as that of a cocktail target in saving wall loss, while the former design not only remarkably simplifies the target fabrication and uses less depleted $U$ material, but also increases the albedo during prepulse, which is good for reducing the hot-spot to wall emission ratio and improving the initial capsule radiation uniformity. 


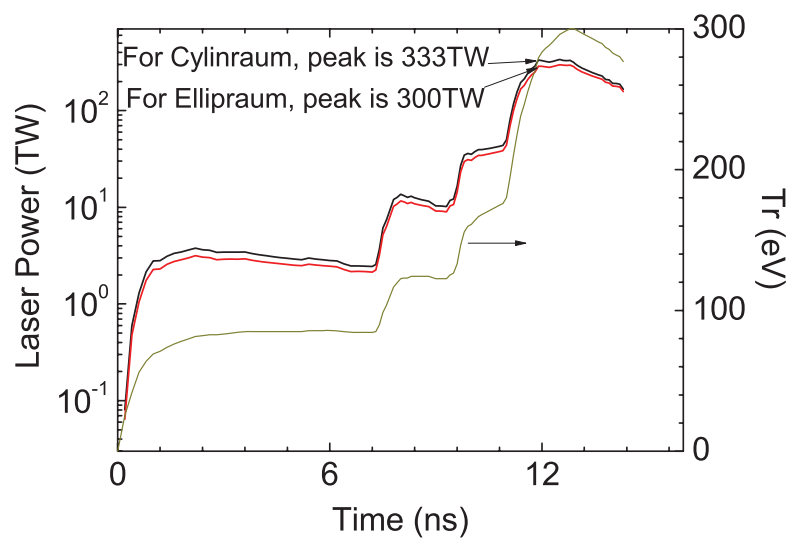

Figure 5. Initial design of pertinent laser power to produce the $300 \mathrm{eV}$ ignition radiation in an ellipraum of $\mathrm{a} / \mathrm{b}=1.6$ or in a cylinraum of $\mathrm{L} / 2 \mathrm{R}=1.84$.

\section{INITIAL DESIGN OF AN ELLIPTICAL HOHLRAUM AND PERTINENT LASER POWER TO PRODUCE A REQUIRED IGNITION RADIATION}

The plasma-filling model $[4,12,13]$ was developed for hohlraum driven by laser with a constant power. In this analytical model, radiation temperature $T_{r}$ in steady-state conditions is related to input laser power P via the power balance:

$$
\eta P=\sigma T_{r}^{4}\left[(1-\alpha) A_{W}+A_{H}\right]
$$

where $\eta$ is the laser-to-X-ray coupling efficiency, $A_{W}$ is the hohlraum wall area, $A_{H}$ is the hohlraum hole area, $\alpha$ is the albedo of wall, and $\sigma$ is the Stefan-Boltzmann constant. The material density inside the hohlraum is obtained by:

$$
\rho=S_{a b l} / V_{h o h l} \times m_{a b l},
$$

where $S_{a b l}$ is the effective area of wall being ablated, $V_{h o h l}$ is the hohlraum volume, and $m_{a b l}$ is mass ablated by radiation. We extend the model to case when a hohlraum is driven by a shaped laser pulse with high contrast $(>1)$ between different steps, which is a typical drive for ignition goal. This extended plasma-filling model, together with 1D simulation to calculate the time-dependent wall albedo under radiation profile, can be used to give an initial design of hohlraum size and pertinent laser to produce a required radiation inside the hohlraum. Certainly, criterion is needed for designing a suitable hohlraum. We define two semi-empirical criterions. One is $n_{e}=0.1$, another one is $n_{I B}=1$. Here, $n_{I B}$ can be defined either as $(R /(\sqrt{2} \sin \delta)) / \lambda_{I B}$ or $\left(\sqrt{2} R_{L E H} / \sin \delta\right) / \lambda_{I B}$, in which $\lambda_{I B}$ is the inverse bremsstrahlung absorption length and $\delta$ is an effective incident angle of laser to hohlraum axis. According to our experience, we usually take $\delta=50^{\circ}$ for ignition design. Under different criterion, it may give a different size of hohlraum. We choose the largest hohlraum as our initial design after considering all the criterions.

As an example, here we use the extended plasma-filling model to give an initial design of an elliptical hohlraum [14], shorted as ellipraum, and pertinent laser to produce a $300 \mathrm{eV}$ ignition radiation [7] in Fig. 5, with a $1 \mathrm{~mm}$ radius capsule inside. We use a and b to denote the long and short lengths of ellipraum, respectively. Shown in Fig. 5 and Fig. 6 are the initial design results of time-dependent laser power, hohlraum size and laser energy in ellipraums of $\mathrm{a} / \mathrm{b}=1.6,1.8$ and 2.0. The initial design of a cylinder hohlraum of $\mathrm{L} / 2 \mathrm{R}=1.84$, shorted as cylinraum, is also given for comparison. As shown, the required laser energy is smaller at a smaller $\mathrm{a} / \mathrm{b}$. It needs $0.83 \mathrm{MJ}$ for $\mathrm{a} / \mathrm{b}=1.6$ ellipraum, more than $10 \%$ lower than for $\mathrm{L} / 2 \mathrm{R}=1.84$ cylinraum which needs $0.93 \mathrm{MJ}$. 


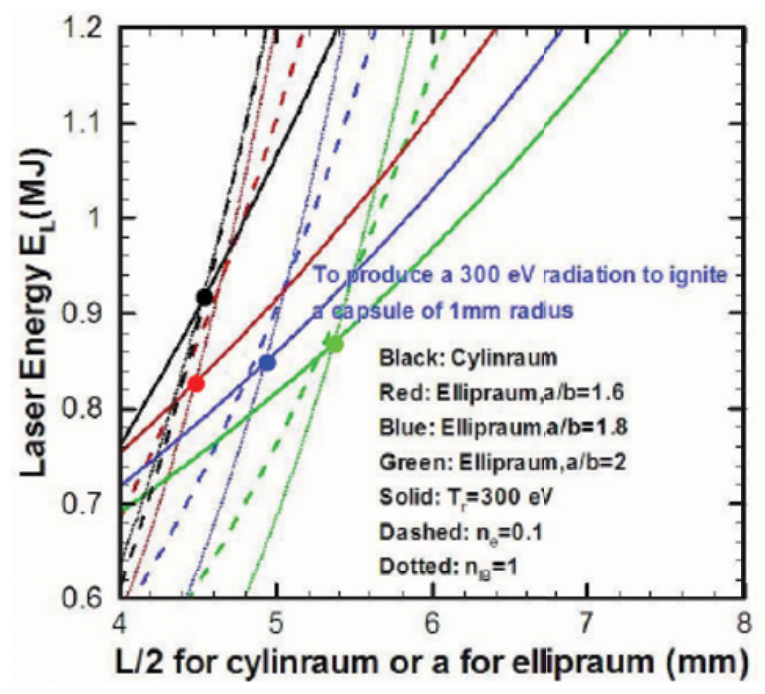

Figure 6. Initial design of laser energy and hohlraum size to produce a $300 \mathrm{eV}$ ignition radiation in ellipraums of $\mathrm{a} / \mathrm{b}=1.6,1.8$ and 2.0 or in a cylinraum of $\mathrm{L} / 2 \mathrm{R}=1.84$.

In fact, the radiation uniformity of an ellipraum also has some superiority to a cylinraum. The coupling between modes in an ellipraum is obviously weaker than in a cylinraum, which benefits to control different modes seperately. Nevertheless, a detail 2D simulation is needed to determine the pointing position and relative powers in the beams for an ellipraum.

\section{SUMMARY}

We have presented some of our recent studies on hohlraum physics. Our simulation results agree with the experimental observations on SGIII laser facility. We had proposed a $0.1 \mu \mathrm{m} \mathrm{Au}+5 \mu \mathrm{m} \mathrm{U}+19.9 \mu \mathrm{m}$ $\mathrm{Au}$ sandwich hohlraum for $300 \mathrm{eV}$ ignition target. Moreover, we have extrapolated the plasma-filling model to the case of hohlraums driven by a shaped laser pulse and applied it to give an initial design of a hohlraum size and pertinent drive laser in order to generate a required radiation. From our initial design, an ellipraum of $\mathrm{a} / \mathrm{b}=1.6$ and $\mathrm{b} / r_{\text {cap }}=2.8$ is superior to an cylinraum of $0.5 \mathrm{~L} / \mathrm{R}=1.6 \sim 1.8$ and $\mathrm{R} / r_{\text {cap }}=2.5$ for $300 \mathrm{eV}$ ignition.

\section{References}

[1] S. Atzeni, and J. Meyer-Ter-Vehn, The Physics of Inertial Fusion. Oxford Science Press, London (2004)

[2] S. Haan, et al., Phys. Plasmas 18, 051001 (2011)

[3] M. D. Rosen, et al., High Energy Density Physics 7, 180 (2011)

[4] M. B. Schneider, et al., Phys. Plasmas 13, 112701 (2006)

[5] W. Y. Huo, et al., Phys. Plasmas 17, 123114 (2010)

[6] X. Li, et al., Laser Part. Beams 28, 75 (2010)

[7] K. Lan, et al., Laser Part. Beams 28, 421 (2010)

[8] Y. Li, et al., Phys. Plasmas 17, 042704 (2010)

[9] Y. Li, W. Y. Huo and K. Lan, Phys. Plasmas 18, 022701 (2011) 
EPJ Web of Conferences

[10] L. J. Suter, Phys. Plasmas 3, 2057 (1996)

[11] L. J. Suter, Phys. Plasmas 7, 2092 (2000); D. A. Callahan, Phys. Plasmas 13, 056307 (2006); O S. Jones, et al., Phys. Plasmas 14, 056311 (2007)

[12] E. L. Dewald, et al., Phys. Rev. Lett. 95, 215004 (2005)

[13] J. W. McDonald, et al., Phys. Plasmas 13, 032703 (2006)

[14] P. Amendt, et al., Phys. Plasmas 14, 056312 (2007); M. Vandenboomgaerde, et al., Phys. Rev. Lett. 99, 065004 (2007) 